\title{
Efficient Computation of the Exponential Operator for Large, Sparse, Symmetric Matrices
}

\author{
Luca Bergamaschi \\ Dipartimento di Metodi e Modelli Matematici per le Scienze Applicate \\ Università di Padova, via Belzoni 7, 35131 Padova, Italy \\ Marco Vianello \\ Dipartimento di Matematica Pura e Applicata \\ Università di Padova, via Belzoni 7, 35131 Padova, Italy
}

\begin{abstract}
In this paper we compare Krylov subspace methods with Chebyshev series expansion for approximating the matrix exponential operator on large, sparse, symmetric matrices. Experimental results upon negative-definite matrices with very large size, arising from (2D and 3D) FE and FD spatial discretization of linear parabolic PDEs, demonstrate that the Chebyshev method can be an effective alternative to Krylov techniques, especially when memory bounds do not allow the storage of all Ritz vectors. We discuss also sensitivity of Chebyshev convergence to extreme eigenvalue approximation, as well as reliability of various a priori and $a$ posteriori error estimates for both methods.
\end{abstract}

KEY WORDS Krylov subspace, Chebyshev series, exponential operator, parabolic PDE

\section{Introduction}

The problem of approximating the matrix exponential operator for large, sparse, symmetric negative-definite matrices, is strictly connected to the numerical solution of $n$-dimensional systems of ODEs like

$$
\dot{\boldsymbol{y}}=-A \boldsymbol{y}+\boldsymbol{b}(t), \quad t>0 ; \quad \boldsymbol{y}(0)=\boldsymbol{y}_{0},
$$

which arise from the space discretization of linear parabolic PDEs (method of lines, linear Galerkin method). 
Indeed, explicit time integration schemes can be constructed without stability restrictions on the time step. These schemes rest on the possibility of efficiently approximating the operator action $\exp (-\tau A) \boldsymbol{v}$, where $\boldsymbol{v} \in \mathbb{R}^{n}$ is a given vector and $\tau>0$ is a scaling factor related to the step size; cf. [9,11]. In this framework, Krylov subspace techniques have been shown to be very effective, since they exhibit remarkable speedups, both in terms of matrix-vector products and total computing time, when compared with the traditional (implicit) time-stepping methods, such as the popular Crank-Nicholson scheme; see for example $[5,6,7,9,11,17]$ and the references therein.

As known, the main idea of Krylov-based methods in computing $f(A) v$ for (analytic) matrix functions, consists of approximately projecting $f(A)$ onto a "small" Krylov subspace $K_{m}=\left\{\boldsymbol{v}, A \boldsymbol{v}, \ldots, A^{m-1} \boldsymbol{v}\right\}$. This reduces the problem to compute $f\left(H_{m}\right)$ in view of the approximation

$$
f(A) \boldsymbol{v} \approx \beta V_{m} f\left(H_{m}\right) \boldsymbol{e}_{1},
$$

where $V_{m}=\left[\boldsymbol{v}_{1}, \boldsymbol{v}_{2}, \ldots, \boldsymbol{v}_{m}\right],\left\{\boldsymbol{v}_{i}\right\}$ being the orthonormal basis of $K_{m}$ and $H_{m}$ the Hessenberg matrix produced by the Arnoldi process with $\boldsymbol{v} /\|\boldsymbol{v}\|_{2}$ as initial vector, $\beta=$ $\|v\|_{2}$. When $A$ is hermitian, $H_{m} \equiv T_{m}$ is tridiagonal, and the iterative process is known as the Lanczos process. The quasi-optimality property of the Krylov subspace approximation keeps the number $m$ reasonably small, being tailored on the spectral structure of $\boldsymbol{v}$ and $A$.

Another polynomial method for the computation of functions of symmetric matrices, is based on the Chebyshev series expansion of the corresponding scalar function on the spectral interval of $A$, say $\left[\lambda_{1}, \lambda_{n}\right]$, assuming $f$ analytic in a neighborhood of such an interval. The approximation of $f(A) v$ takes on the form

$$
f(A) \boldsymbol{v} \approx \boldsymbol{s}_{m}=S_{m}(B) \boldsymbol{v}=\sum_{k=0}^{m} a_{k} C_{k}(B) \boldsymbol{v}
$$

where $C_{k}$ is the $k^{t h}$ Chebyshev polynomial of the first kind,

$$
B=\frac{1}{\ell_{1}} A-\frac{\ell_{1}}{\ell_{2}} I, \quad \ell_{1}=\left(\lambda_{n}-\lambda_{1}\right) / 2 \text { and } \ell_{2}=\left(\lambda_{n}+\lambda_{1}\right) / 2
$$

and the $a_{k}$ are the Chebyshev coefficients of $f\left(\ell_{1} t+\ell_{2}\right), \quad t \in[-1,1]$. In view of the threeterm recurrence for the Chebyshev polynomials, the expansion in (1.3) can be conveniently implemented at the main cost per step of a matrix-vector product and without any scalar product. Chebyshev polynomial approximation for the exponential operator was originally considered in [5,20] for the symmetric case. Recently, a non-symmetric analogous based on Faber polynomials and Faber series has been studied in [15].

The number of iterations and the overall computational cost of Chebyshev method is usually larger than Krylov's, since the former method is thought to approximate the matrix $f(A)$ and cannot conveniently exploit the spectral structure of $\boldsymbol{v}$. However, if we take into account computer storage, Chebyshev method requires only six vectors (in addition to $A$ and $\boldsymbol{v}$ ), while the standard Krylov process needs to keep in memory all the $m$ Ritz vectors. For this reason, Krylov method has to be somehow modified on very large size problems. A straightforward idea, which we adopt in this paper, is that of computing first the vector $\boldsymbol{c}=\|\boldsymbol{v}\|_{2} f\left(T_{m}\right) \boldsymbol{e}_{1}$ storing only $\boldsymbol{v}_{1}, \ldots, \boldsymbol{v}_{\nu}, \nu<m$ depending on the actual available memory. Clearly, reliable a priori or a posteriori error estimates have to be used to evaluate the final iteration number $m$. The second step consists of restarting the Lanczos process from $\boldsymbol{v}_{\nu+1}$ up to $\boldsymbol{v}_{m}$ without storing them, and computing the right hand side of (1.2) as a 
linear combination $\sum_{i=1}^{m} c_{i} \boldsymbol{v}_{i}$. Note that the computing time increases up to twice that of the standard process.

In the present paper we propose an efficient implementation of the Chebyshev method for the exponential operator, based on control of the relative error, as described in $\S 2$. The key tools are: an efficient approximation of the spectral interval using Lanczos method for the largest eigenvalue [10] and a Rayleigh Quotient - based technique for the smallest [2], use of both a priori and a posteriori convergence estimates, and a lower bound of $\|\exp (-\tau A) \boldsymbol{v}\|_{2}$ based on the computation of an eigenvector associated to $\lambda_{1}$. In $\S 3$ the implementation issues of two-step Krylov algorithm are discussed.

In $\S 4$ we compare the Krylov and Chebyshev methods on very large size matrices arising from both Finite Difference (FD) and Finite Element (FE) space discretization of 2D and 3D linear parabolic PDEs. Numerical experiments show that Krylov method always requires less iterations than Chebyshev method, as expected. In particular, in the FD case the ratio between Chebyshev and Krylov iterations ranges from about 1 to 1.7, depending on the scaling factor $\tau$. However, for large problems, the need of running twice the Lanczos process makes Chebyshev method more convenient in terms of total computing time. In the FE test problems the ratio of iteration numbers is always less than 1.2, and so the computing times of the two methods are very close already for "small" problems. Hence, when Krylov has to be doubled, Chebyshev method becomes even more convenient than in FD problems (showing a maximum speedup close to 2). We give also evidence of the low sensitivity of Chebyshev convergence to the smallest eigenvalue approximation, which could be even estimated on a coarser mesh, when available. On the other hand, the largest eigenvalue can be computed at a price of a few matrix-vector products by Lanczos process, making the cost of this preprocessing stage a small percentage of the overall cost. Finally, reliability of various a priori and a posteriori error estimates available in the literature, is discussed for both methods. Some concluding remarks and possible developments are given is $\S 5$.

\section{Chebyshev series approach}

One of the main features in using Chebyshev series is the computation of the Chebyshev coefficients $a_{k}$, cf. (1.3), which depend on the scalar function $f$, and the spectral interval of $A$. In the case of the exponential function $f(\lambda)=\exp (-\tau \lambda), \lambda \in\left[\lambda_{1}, \lambda_{n}\right], \tau>0$, it is known that the coefficients are related to modified Bessel functions $I_{k}$ [1, §9.6]. More precisely,

$$
a_{0}=\exp \left(-\tau \ell_{2}\right) I_{0}\left(-\tau \ell_{1}\right), \quad a_{k}=2 \exp \left(-\tau \ell_{2}\right) I_{k}\left(-\tau \ell_{1}\right)
$$

where the $\ell_{i}$ 's are defined in $\S 1$; cf. [14, $\left.\S 6.5\right]$. A sharp approximation (from outside) of the spectral interval is therefore required, which represents a drawback of Chebyshev method with respect to Krylov method. In practice, however, the cost of this approximation on very large size problems can be made small, when compared with the overall cost. This is obtained by the following approach: the largest eigenvalue is computed by the Lanczos process, at the price of a few matrix-vector multiplications [10], while the smallest can be conveniently approximated with a diagonally-preconditioned conjugate gradient technique, applied to the minimization of the Rayleigh Quotient, as in [2]. It is worth noting that both these procedures provide (monotone) approximations from the inside of the spectral interval, while convergence of Chebyshev series is guaranteed if an estimate from outside 
is at hand. We had also experimental evidence of the bad behavior of the algorithm even when the approximation from inside are accurate. The problem of approximating $\left[\lambda_{1}, \lambda_{n}\right]$ from outside, however, can be easily solved, exploiting the linear and monotone convergence character of both methods.

The well known three-term recurrence for the modified Bessel functions $I_{k}$ cannot be used forward, in view of its instability when applied to arguments with large absolute value. This problem can be circumvented using backward the three-term recurrence, i. e.

$$
I_{k-2}(z)=\frac{k}{2 z} I_{k-1}(z)+I_{k}(z) \quad k=m, m-1, \ldots, 2
$$

once a realistic a priori upper bound of the required number $m$ of coefficients is available. In fact, the two starting values $I_{m}$ and $I_{m-1}$ of the recurrence can be easily computed at a high accuracy by a well-known asymptotic expansion [1, $\S 9.7 .1]$. Such an a priori upper bound can be obtained starting from the absolute error estimate in the euclidean norm:

$$
\begin{aligned}
\epsilon_{m}^{C h e b}=\left\|\exp (-\tau A) \boldsymbol{v}-\sum_{k=0}^{m} a_{k} C_{k}(B) \boldsymbol{v}\right\| & =\left\|\sum_{k=m+1}^{\infty} a_{k} C_{k}(B) \boldsymbol{v}\right\| \leq \beta \sum_{k=m+1}^{\infty}\left|a_{k}\right| \leq \\
& \leq 2 \beta \exp \left(-\tau \lambda_{1}\right) E(m),
\end{aligned}
$$

with $B$ is defined in (1.4), $a_{k}$ in (1.3), and $\beta=\|v\|$,

$$
E(m)= \begin{cases}\exp \left(\frac{-b(m+1)^{2}}{\tau \lambda_{n}}\right)\left[1+\sqrt{\frac{\tau \lambda_{n} \pi}{4 b}}\right]+\frac{d^{\tau \lambda_{n}}}{1-d} & \text { if } \quad m \leq \tau \lambda_{n} \\ \frac{d^{m}}{1-d} & \text { if } \quad m>\tau \lambda_{n}\end{cases}
$$

where $b \approx 0.618, d \approx 0.438$; these values, together with (2.7) are taken from [19]. Whenever a lower bound of $\|\exp (-\tau A) \boldsymbol{v}\|$ is available, we can estimate the number $m=m(\varepsilon)$ such that the relative error is less than a prescribed tolerance $\varepsilon$. A lower bound of such a norm can be obtained by writing $\boldsymbol{v}$ as a linear combination of the orthonormal eigenvectors $\boldsymbol{u}_{1}, \ldots, \boldsymbol{u}_{n}$ of $A, \boldsymbol{v}=\sum_{i=1}^{n} c_{i} \boldsymbol{u}_{i}$, so that

$$
\|\exp (-\tau A) \boldsymbol{v}\|=\sqrt{c_{1}^{2} e^{-2 \tau \lambda_{1}}+\ldots+c_{n}^{2} e^{-2 \tau \lambda_{n}}} \geq\left|c_{1}\right| e^{-\tau \lambda_{1}}
$$

Since the Rayleigh Quotient minimization yields also the eigenvector $\boldsymbol{u}_{1}$, the coefficient $c_{1}$ can be easily computed taking into account the orthonormality of the eigenvectors of $A$ :

$$
\boldsymbol{u}_{1}^{T} \boldsymbol{v}=\boldsymbol{u}_{1}^{T} \sum_{i=1}^{n} c_{i} \boldsymbol{u}_{i}=c_{1} \boldsymbol{u}_{1}^{T} \boldsymbol{u}_{1}=c_{1}
$$

We can now write the following estimate of the relative error:

$$
\frac{\epsilon_{m}^{C h e b}}{\|\exp (-\tau A) \boldsymbol{v}\|} \leq \frac{2 \beta}{\left|c_{1}\right|} E(m),
$$

(cf. (2.7)), thus obtaining $m(\varepsilon)=\min \left\{m: E(m)<\left|c_{1}\right| \varepsilon /(2 \beta)\right\}$.

Another estimate for the absolute error was originally proposed in [5], i. e.,

$$
\epsilon_{m}^{C h e b} \leq\left[\sqrt{2 \pi}+O\left(\frac{1}{a}\right)\right] \frac{\sqrt{a}}{m} \exp \left[-\frac{m}{a^{2}}+O\left(\frac{m^{4}}{a^{3}}\right)\right], \quad m \leq a,
$$


where we set $a=\tau \lambda_{n} / 2$. Although (2.10) be asymptotically sharper than (2.6)-(2.7), it is meaningful only for "large" $a$ and $\sqrt{a} \ll m \ll a$. For this reason, and in view of the fact that at large dimension the cost of precomputation of Chebyshev coefficients is really negligible, we chose the bound (2.7) in our implementation.

At this point, once the first $m(\varepsilon)$ Chebyshev coefficients have been computed, using backward the Bessel recurrence as described above, we can start the evaluation of the sum in (1.3) as:

$$
\boldsymbol{s}_{k+1}=\boldsymbol{s}_{k}+a_{k+1} \boldsymbol{v}_{k+1},
$$

where $\boldsymbol{v}_{k}=C_{k}(B) \boldsymbol{v}$ satisfies the three-term recurrence:

$$
\boldsymbol{v}_{k+1}=2 B \boldsymbol{v}_{k}-\boldsymbol{v}_{k-1}, \quad k=1,2, \ldots
$$

Our stopping criterion is not based on the upper bound (2.9), which is aimed only at computing a sufficient number of Chebyshev coefficients. A sharper estimate of the absolute error (the left hand side in (2.6)), can be a priori obtained from:

$$
\epsilon_{m}^{C h e b}=\left\|\sum_{k=m+1}^{\infty} a_{k} C_{k}(B) v\right\| \leq \beta \sum_{k=m+1}^{\infty}\left|a_{k}\right| \approx \beta \sum_{k=m+1}^{m(\varepsilon)}\left|a_{k}\right| .
$$

Since we are interested in the relative error, we also need a reliable approximation of $\|\exp (-\tau A) \boldsymbol{v}\|$ : the latter is easily provided by computing $\left\|\boldsymbol{s}_{k}\right\|$ every $p$ values of the iteration index $k$, where $p$ is a user-defined "checking step", with the following acceptance criterion:

$$
\left\|s_{k_{0}}\right\| \approx\|\exp (-\tau A) \boldsymbol{v}\| \quad \text { as soon as } \quad\left|\frac{\left\|s_{k_{0}}\right\|}{\left\|s_{k_{0}-p}\right\|}-1\right|<0.1,
$$

i. e., as soon as the order of magnitude of $\left\|s_{k}\right\|$ stabilizes. We therefore stop the iterative process (2.12) at the smallest index $m>k_{0}$ such that

$$
\frac{\beta \sum_{k=m+1}^{m(\varepsilon)}\left|a_{k}\right|}{\left\|\boldsymbol{s}_{k_{0}}\right\|} \leq \varepsilon
$$

The Chebyshev series approach is then implemented by the following:

\section{Algorithm: ChebysheV}

1. INPUT: $\varepsilon, \boldsymbol{v}$, the checking step $p, \tau$.

2. Estimate $\lambda_{1}, \lambda_{n}$, by Rayleigh Quotient and Lanczos procedures.

3. Compute the max \# of iterations $m(\varepsilon)$ by (2.9).

4. Compute the first $m(\varepsilon)$ Chebyshev coefficient, by backward Bessel recursion.

5. Compute matrix $B$ as in (1.4), and set $\boldsymbol{v}_{0}:=\boldsymbol{v}, \boldsymbol{v}_{1}:=B \boldsymbol{v}, \boldsymbol{s}_{1}:=a_{1} \boldsymbol{v}_{1}+\frac{a_{0}}{2} \boldsymbol{v}_{0}$.

6. exit_test $:=$ false, norm_test $:=$ false, $k:=1 ; m:=m(\varepsilon)$.

7. WHILE (NOT exit_test) DO

$$
\begin{aligned}
& \boldsymbol{v}_{k+1}:=2 \boldsymbol{B} \boldsymbol{v}_{k}-\boldsymbol{v}_{k-1} \\
& \boldsymbol{s}_{k+1}:=\boldsymbol{s}_{k}+a_{k+1} \boldsymbol{v}_{k+1} \\
& \text { IF }(k \bmod p)=0 \text { AND (NOT norm_test) THEN } \\
& \quad r:=\frac{\left\|\boldsymbol{s}_{k+1}\right\|}{\left\|\boldsymbol{s}_{k+1-p}\right\|}
\end{aligned}
$$




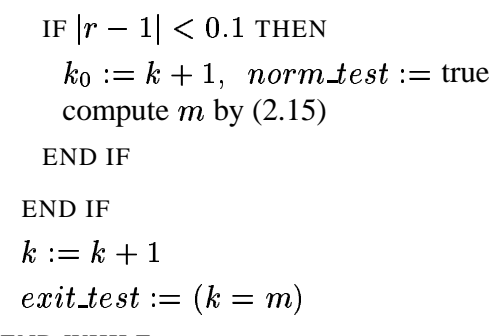

END WHILE

\section{Krylov subspace approach}

Krylov-based method for computing $f(A) v$ has become the most popular, thanks to the following reasons: it is tailored on the spectral structure of both $A$ and $v$, thus achieving a faster convergence than Chebyshev, without requiring any a priori knowledge of the spectral interval; it works for general (non symmetric) matrices provided that the Arnoldi process is used. As for the exponential operator, we mention a Software Package recently developed, named EXPOKIT [18], which implements Krylov based routines for both dense and sparse matrix exponential computation.

The main drawback of the Krylov approach stems from the storage requirement: all the $m$ Lanczos vectors should be kept in memory to get the final approximation of $\exp (-\tau A) \boldsymbol{v}$. As sketched in the Introduction, a simple way to overcome this problem is to run twice the Lanczos process. The first step aims to evaluate the coefficients of (1.2), i. e. the vector $\boldsymbol{c}_{m}=\beta \exp \left(-\tau T_{m}\right) \boldsymbol{e}_{1}$, by keeping in memory only the $\boldsymbol{v}_{1}, \ldots, \boldsymbol{v}_{\nu}, \nu$ depending on actual storage limitations. The second step generates again the Lanczos vectors (starting form the $(\nu+1) t h)$, updating the linear combination as in (1.2). The cost of the first run is generally higher than that of the second one, and depends essentially on the ratio $\nu / m$ : in particular the number of matrix-vector products is reduced in the second step by a factor $1-\nu / m$. Moreover, every iteration in the second run of the Lanczos process can be performed without computing the two scalar products.

Note that in the first run the computation of $\exp \left(-\tau T_{m}\right) \boldsymbol{e}_{1}$ is required for the final tridiagonal matrix $T_{m}$, and, possibly, for some intermediate matrices $T_{k}$, with $k<m$, when the stopping criterion is based on an a posteriori bounds [17]. If the dimension of the Krylov subspace $m$ is not too large, this step can be performed using standard methods; in our applications this number can be of order of the hundreds, and hence ad hoc techniques are required. In $[8,9,17]$, e. g., the use of rational Chebyshev approximation is proposed, while our implementation makes use of the Chebyshev series method, as described in $\S 2$. On large size problems, the cost of this operation turns out to be "negligible" when compared to that of the whole algorithm.

We sketch now the implementation of the first step of the Krylov algorithm, recalling that the Krylov subspace approximation of the exponential operator is given by:

$$
\exp (-\tau A) \boldsymbol{v} \approx \beta V_{m} \exp \left(-\tau T_{m}\right) \boldsymbol{e}_{1}=V_{m} \boldsymbol{c}_{m},
$$

where the tridiagonal matrix $T_{m}$ is defined in terms of the Lanczos coefficients as:

$$
T_{m}=\operatorname{tridiag}\left[\beta_{k}, \alpha_{k}, \beta_{k+1}\right], \quad k=1, \ldots, m .
$$


A reliable estimate of $\epsilon_{m}^{\text {Kryl }}=\left\|\exp (-\tau A) \boldsymbol{v}-V_{m} \boldsymbol{c}_{m}\right\|$ is required to stop the iterative process. In principle, it could be a priori estimated using (2.7) or (2.10), since $\epsilon_{m}^{\text {Kryl }} \leq$ $\epsilon_{m}^{C h e b}$, i. e. the error of Krylov approximation is bounded by that of Chebyshev polynomial approximation. In [11] another a priori estimate for the Krylov process is obtained, without resorting to Chebyshev polynomials, namely

$$
\epsilon_{m}^{K r y l} \leq\left(3 \frac{\tau \lambda_{n}}{m^{2}}+2 \frac{\sqrt{\lambda_{n} \tau}}{m}\right) \exp \left(-\beta m^{2} /\left(\tau \lambda_{n}\right)\right) .
$$

Numerical experience, however, shows that all the estimates above are rather pessimistic, and hence cannot be used in practice. For these reasons, the stopping criterion for the first Lanczos run is based on the a posteriori absolute error estimate

$$
\epsilon_{m}^{K r y l} \leq \operatorname{err}(m)=\beta_{m+1}\left|\boldsymbol{e}_{m}^{T} \phi\left(-\tau T_{m}\right) \boldsymbol{e}_{1}\right| \beta, \quad \phi(z)=\frac{\exp (z)-1}{z} .
$$

Such an estimate was first proposed by Saad [17], with a derivation which is plausible only for small $\tau \lambda_{n}$. In [12] the reliability of this bound has been shown also in the general case.

The evaluation of (3.18) is performed every $p$ iterations via the following steps. First, we compute

$$
\boldsymbol{c}_{k}=\beta \exp \left(-\tau T_{k}\right) \boldsymbol{e}_{1},
$$

by the Chebyshev algorithm described in $\S 2$. In order to get an a posteriori bound of the relative error, an estimate of $\|\exp (-\tau A) \boldsymbol{v}\|$ can be easily obtained by $\left\|\boldsymbol{c}_{\boldsymbol{k}}\right\|$ in view of the identity (valid at least in exact arithmetic):

$$
\left\|V_{k} \boldsymbol{c}_{k}\right\|^{2}=\boldsymbol{c}_{k}^{T} V_{k}^{T} V_{k} \boldsymbol{c}_{k}=\boldsymbol{c}_{k}^{T} \boldsymbol{c}_{k}=\left\|\boldsymbol{c}_{k}\right\|^{2} .
$$

Our numerical experiments show that the loss of orthogonality due to round-off errors does not significantly affect the order of magnitude of $\left\|V_{k} c_{k}\right\| ;$ cf. also [4]. As in the previous algorithm, we adopt the acceptance criterion

$$
\left\|\boldsymbol{c}_{k_{0}}\right\| \approx\|\exp (-\tau A) \boldsymbol{v}\| \quad \text { as soon as } \quad\left|\frac{\left\|\boldsymbol{c}_{k_{0}}\right\|}{\left\|\boldsymbol{c}_{k_{0}-p}\right\|}-1\right|<0.1,
$$

whose meaning is the same of (2.14). The first Lanczos run is then stopped at the smallest $(\bmod p)$ index $m>k_{0}$ such that

$$
\frac{e r r(m)}{\left\|c_{k_{0}}\right\|}<\varepsilon
$$

where, taking into account that

$$
\operatorname{err}(k)=\frac{\beta_{k+1}}{\tau}\left|\boldsymbol{e}_{k}^{T} T_{k}^{-1}\left(\boldsymbol{c}_{k}-\beta \boldsymbol{e}_{1}\right)\right|,
$$

we solve the tridiagonal system $T_{k} \boldsymbol{y}=\boldsymbol{c}_{k}-\beta \boldsymbol{e}_{1}$, and finally compute, every $p$ iterations, $\operatorname{err}(k)=\tau^{-1} \beta_{k+1}\left|y_{k}\right|$.

We do not report the pseudocode of the well-known Krylov algorithm, the second run being trivially implemented as described above. 


\section{Numerical Results}

Our purpose is to demonstrate the effectiveness of the Chebyshev approach with respect to the Krylov technique, on problems of very large size. Consider the linear diffusion problem with mixed boundary conditions, like:

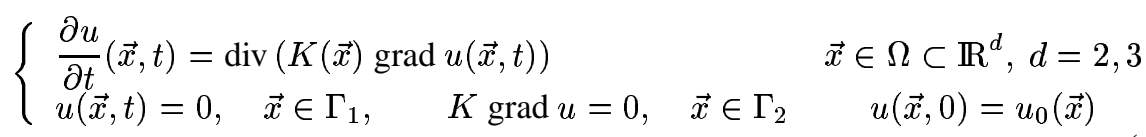

where $\Gamma_{1} \cup \Gamma_{2}=\partial \Omega$.

This section is devoted to the comparison of the two methods on test cases arising from space discretization of (4.24) by Finite Differences, on the unitary cubic domain, and by Finite Elements on 2D and 3D rectangular domains. All the computations were made on an Alpha station 600Mhz with $128 \mathrm{Mb}$ RAM. The maximum number $\nu$ of storable Ritz vectors for the Krylov algorithm is a priori estimated taking into account this limitation. The total CPU times reported in the tables below do not take into account the computation of $\boldsymbol{c}_{\boldsymbol{k}}$ in (3.19) as for Krylov algorithm, whereas they are comprehensive of eigenvalue estimation in the Chebyshev case.

\subsection{D Finite Difference Matrices}

Consider (4.24) with $K=I$, and $\Gamma_{1}=\partial \Omega$ (purely Dirichlet boundary conditions). Discretization of the Laplace operator by Finite Differences with constant step size $h=1 / s$ gives raise to a $n\left(=s^{3}\right)$-dimensional system of the form (1.1), (with $\boldsymbol{b} \equiv \mathbf{0}$ ) where $A$ is a symmetric positive definite eptadiagonal matrix. We choose the initial vector to be $\boldsymbol{v}=\boldsymbol{y}_{0}=[1,1, \ldots, 1]^{T}$. Four different grids were considered with size $s=40,60,80$ and 100, respectively. The extreme eigenvalues of the respective matrices are reported in Table 1. For this case we have used as an approximation of $\lambda_{1}^{(s)}$ the value of $\lambda_{1}$ on a coarser mesh, since the smallest eigenvalue of the discrete problem converges, as $s$ tends to infinity, to the smallest eigenvalue of the continuous problem.

For our numerical tests it was sufficient to calculate the smallest eigenvalue $\lambda_{1}^{(10)}$ on the $10^{3}$ grid, with a good saving of the computing time. As for the largest eigenvalue instead of using the costly Lanczos method we simply took the infinity norm of the matrix $A$ as an upper bound. The Table accounts for the reliability of this approximation. We

\begin{tabular}{rrccc}
$s$ & d.o.f. & $\lambda_{1}^{(s)}$ & $\lambda_{n}^{(s)}$ & $\left\|A_{s}\right\|_{\infty}$ \\
\hline 10 & 1,000 & $.24305 \mathrm{e}+02$ & $.11884 \mathrm{e}+04$ & $.12000 \mathrm{e}+04$ \\
$\ldots$ & $\ldots$ & $\ldots$ & $\ldots$ & $\ldots$ \\
40 & 64,000 & $.28168 \mathrm{e}+02$ & $.19087 \mathrm{e}+05$ & $.19200 \mathrm{e}+05$ \\
60 & 216,000 & $.28640 \mathrm{e}+02$ & $.43086 \mathrm{e}+05$ & $.43200 \mathrm{e}+05$ \\
80 & 512,000 & $.28879 \mathrm{e}+02$ & $.76692 \mathrm{e}+05$ & $.76800 \mathrm{e}+05$ \\
100 & $1,000,000$ & $.29023 \mathrm{e}+02$ & $.11988 \mathrm{e}+06$ & $.12000 \mathrm{e}+06$
\end{tabular}

Table 1. Extreme eigenvalues for the Finite Difference matrices with various grid sizes. 


\begin{tabular}{|c|c|c|c|c|c|c|c|c|}
\hline \multirow[b]{3}{*}{$s$} & \multirow[b]{3}{*}{$\tau$} & \multirow[b]{3}{*}{$\lambda_{n}\left(\tau A_{s}\right)$} & \multicolumn{4}{|c|}{ Lanczos } & \multirow{2}{*}{\multicolumn{2}{|c|}{ Chebyshev }} \\
\hline & & & & & $\mathrm{CP}$ & (s) & & \\
\hline & & & $\nu$ & \# it. & 1 st run & total & \# it. & CPU (s) \\
\hline \multirow[t]{3}{*}{40} & 0.01 & 191 & 200 & $\overline{53}$ & 2.64 & 2.64 & 58 & 2.48 \\
\hline & 0.03 & 573 & 200 & 83 & 4.16 & 4.16 & 100 & 4.05 \\
\hline & 0.10 & 1,909 & 200 & 108 & 5.42 & 5.42 & 182 & 7.40 \\
\hline \multirow[t]{3}{*}{60} & 0.01 & $\overline{431}$ & $\overline{53}$ & 81 & 14.97 & 19.46 & 86 & 14.17 \\
\hline & 0.03 & 1,293 & 53 & 125 & 22.44 & 33.95 & 148 & 24.38 \\
\hline & 0.10 & 4,309 & 53 & 161 & 28.51 & 45.72 & 270 & 44.90 \\
\hline \multirow[t]{3}{*}{80} & 0.01 & 767 & 8 & 108 & 48.78 & 89.42 & 115 & 50.34 \\
\hline & 0.03 & 2,301 & 8 & 167 & 75.13 & 139.67 & 199 & 88.05 \\
\hline & 0.10 & 7,670 & 8 & 214 & 96.14 & 179.80 & 365 & 160.79 \\
\hline \multirow[t]{3}{*}{100} & 0.01 & 1,199 & 2 & 136 & 118.45 & 224.57 & 144 & 115.43 \\
\hline & 0.03 & 3,396 & 2 & 207 & 180.10 & 341.40 & 249 & 200.18 \\
\hline & 0.10 & 11,989 & 2 & 267 & 232.41 & 441.54 & 455 & 366.67 \\
\hline
\end{tabular}

Table 2. Number of iterations and CPU time for the Lanczos and Chebyshev algorithms applied to the FD sample problems with $\epsilon=10^{-8}$.

have compared the two polynomial algorithms described in the previous sections in the computation of $\exp \left(-\tau A_{s}\right) \boldsymbol{v}$ for three values of $\tau=0.01,0.03,0.1$.

Table 2 shows the results of the simulations in terms of number of iterations and computing time for both the procedures. The implementation of the Krylov algorithm, starting from the $s=60$ case, could not store all the Ritz vectors within the available memory, and hence requires a certain number of iterations in the second run. The Chebyshev code, however, could run using less than $128 \mathrm{Mb}$ RAM also when addressing the largest problem (with $s=100$ ).

Figure 1 displays the convergence profiles for the intermediate $s=60$ mesh. From this plot and Table 2 we can conclude that the Chebyshev algorithm becomes competitive with Krylov procedure either when the spectral radius of the matrix is not too large $(\tau=0.01$ case), or when the size of the problem is sufficiently large to require the execution of the second Lanczos run $(s=80,100)$. Observe, however, that the CPU time scales better with $\tau$ for the Krylov algorithm rather than for the Chebyshev method. This behavior suggests that, at very large $\rho(\tau A)$, the Krylov approach again could perform better than Chebyshev, even taking into account its second run.

\subsection{Sensitivity of Chebyshev convergence to eigenvalue approximation}

The need of estimating the extreme eigenvalues of $A$ is commonly considered a weak point of the Chebyshev polynomial approximation [9]. First, if the spectral interval is approximated from inside, convergence is not guaranteed, as confirmed by our numerical experiments. This difficulty can be circumvented by slightly modifying the basic procedures (Rayleigh Quotient for the smallest, Lanczos for the largest) in order to obtain approximations on the safe side. Moreover, accurate computation of the smallest eigenvalue is costly because it requires the solution of linear systems (when using Lanczos method), or several 


\section{Chebychev vs Krylov}

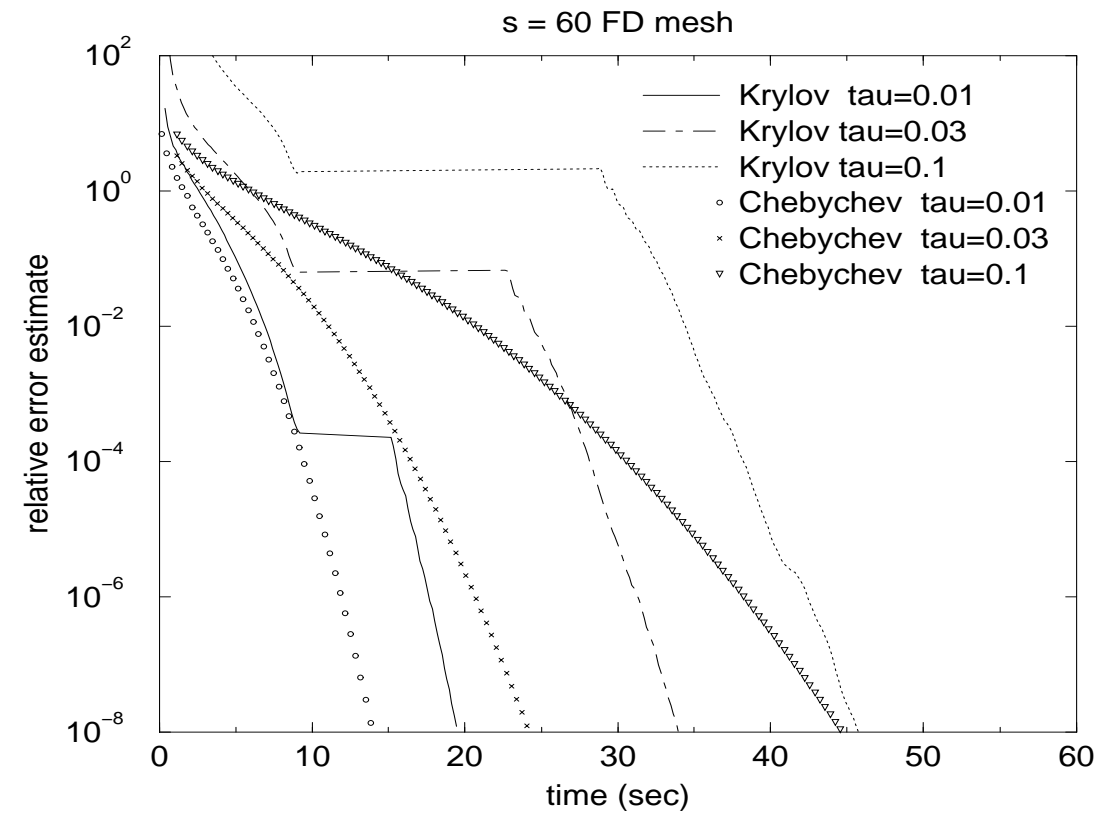

Figure 1. Relative error norm versus CPU time in the evaluation of $\exp (-\tau A) \boldsymbol{v}$ for the $60^{3}$ mesh and for the three different values of $\tau$. The horizontal lines account for the computation of the Lanczos coefficients (from $\nu+1$ to $m$ ) in the first Lanczos run.

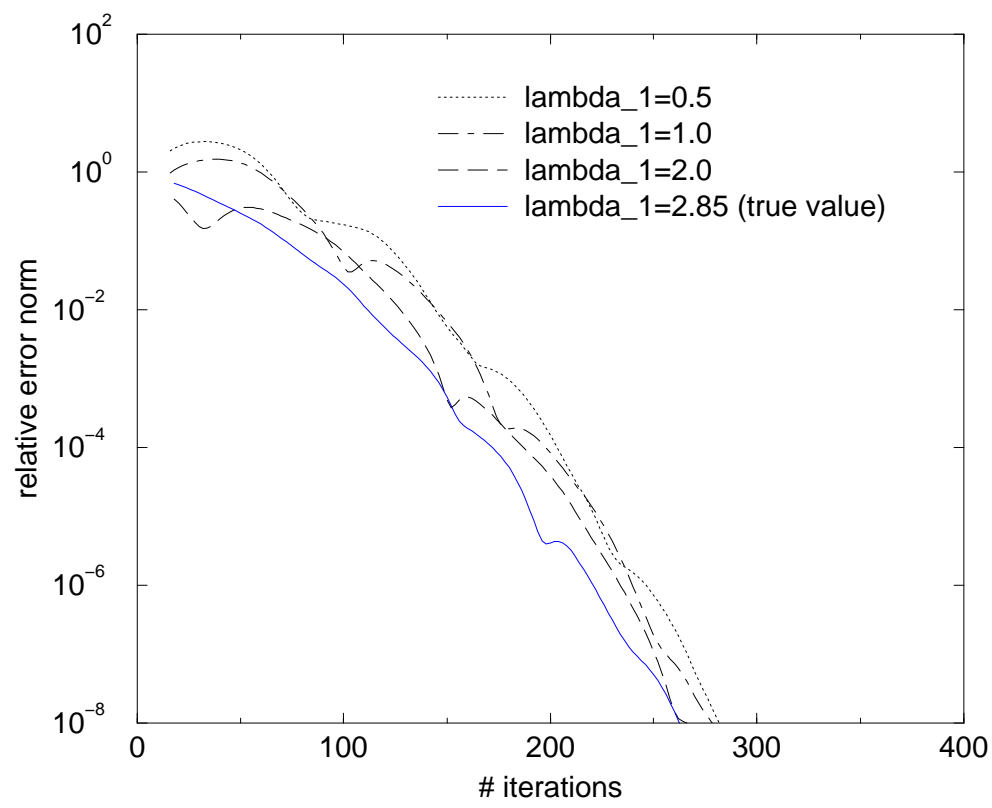

Figure 2. Sensitivity of Chebyshev convergence to the approximation of the smallest eigenvalue $\lambda_{1}$ : FD test case with $s=60$ and $\tau=0.1$. 


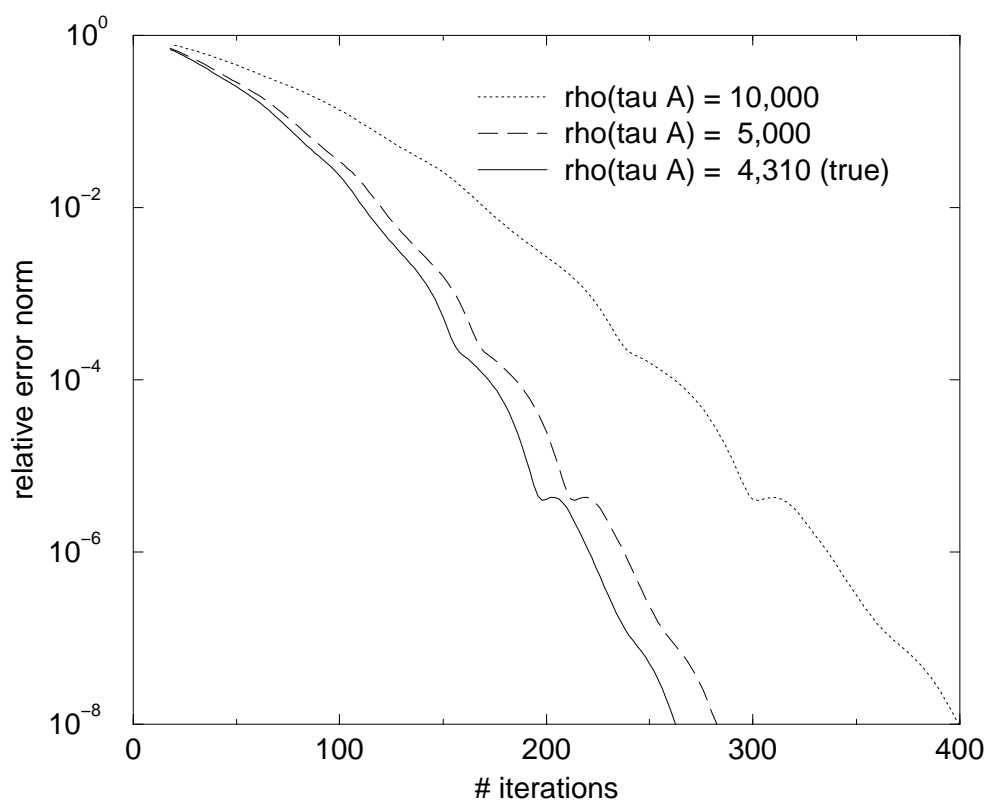

Figure 3. Sensitivity of Chebyshev convergence to the approximation of the largest eigenvalue $\lambda_{n}=\rho(\tau A):$ FD test case with $s=60$ and $\tau=0.1$.

matrix-vector products (if the Rayleigh Quotient minimization is employed). However Figure 2 shows that the convergence rate of the Chebyshev approximation is not very sensitive to the accuracy in approximating $\lambda_{1}$. Thus in practice, the Rayleigh Quotient minimization can be stopped with a rough tolerance (say 50\%) at the price of a few matrix-vector products. When possible, one could even resort to a coarser mesh to approximate the smallest eigenvalue in a much cheaper way.

On the contrary Figure 3 makes clear that the largest eigenvalue has to be more accurately estimated to prevent a severe deterioration of the convergence. It is worth noting, however, that again a few matrix-vector products yield $\lambda_{n}$ to a sufficient accuracy (say 15\%). This argument applies particularly in the Finite Element case (see §4.4.), where the infinity norm does not provide a good approximation of the spectral radius of $A$. We stress finally that, in all our numerical experiments, estimation of the spectral interval ranged from $1 \%$ to at most $15 \%$ of the overall cost of Chebyshev algorithm, depending on the spectral radius of $\tau A$. Moreover, if $\exp (-\tau A) \boldsymbol{v}$ has to be computed for different $\tau$ and $\boldsymbol{v}$, as should be the case in the solution of (1.1), the extreme eigenvalues of $A$ can be approximated once and for all.

\subsection{Reliability of error estimates}

In this section, for both Chebyshev and Krylov algorithms developed in $\S 2$ and $\S 3$, we compare "true" relative errors with a priori and a posteriori relative error estimates, on the previous FD test case with $s=60$. In Figures 4, 5, 6 we plotted the three a priori estimates provided by Stewart and Leyk (2.6)-(2.7), Druskin and Knizhnermann (2.10), 


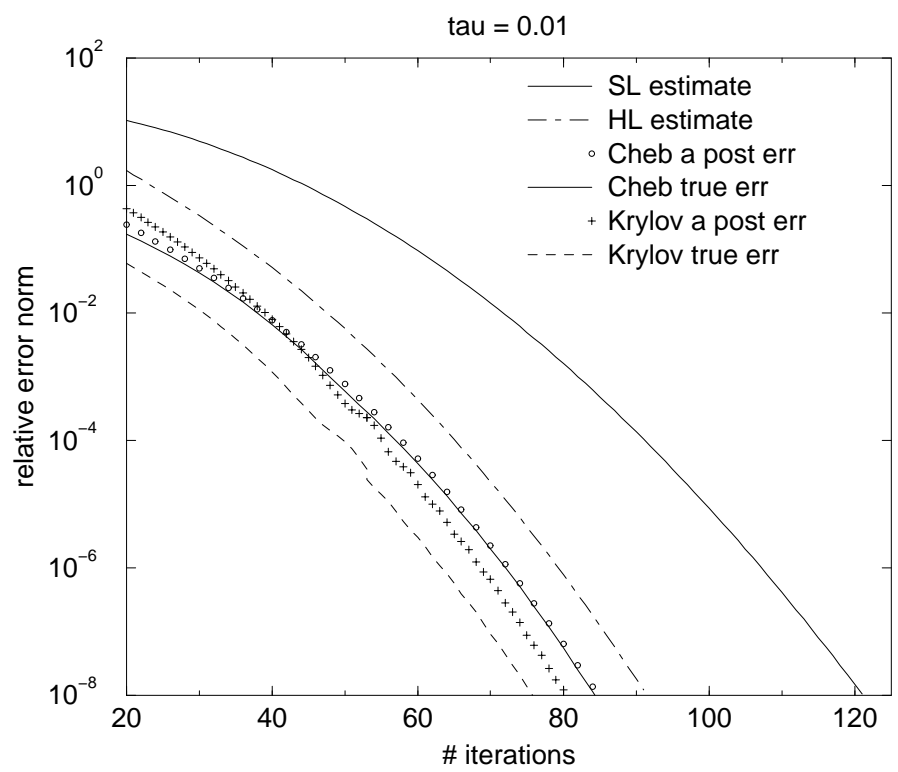

Figure 4. Comparison of Chebyshev and Krylov true errors with a priori and a posteriori estimates for the FD case with $s=60$ and $\tau=0.01$.

and by Hochbruch and Lubich (3.17), named SL, DK and HL, respectively (all divided by $\|\exp (-\tau A) \boldsymbol{v}\|)$.

These estimates have been developed by the respective authors to bound the error of Krylov approximation, the first two being applicable also to Chebyshev approximation. From Figures 4, 5, 6 we can notice that estimate HL also bounds the true error of the Chebyshev approximation. This is not surprising, as this estimate can be derived via Faber series (cf. [11, §5], [13]) which are strictly related to Chebyshev series in the symmetric case. Comparison of these three profiles with Krylov and Chebyshev true error plots shows that they are rather pessimistic and cannot be used in practice, especially for Krylov algorithm. Bound (2.10) turns out to be asymptotically the sharpest one (see e. g. Figure 6); however, as already observed in $\S 2$, its application is meaningful only for "large" $\tau \lambda_{n}$ and $m \ll \tau \lambda_{n}$. This is not the case of the example in Figure 4, where $\tau \lambda_{n}=439$, and $m>70$ is already required to get a relative error below $10^{-4}$ : hence the plot of bound (2.10) is not provided in Figure 4. We note that the a posteriori bound (2.15) we use for Chebyshev algorithm is very sharp, while the relative error estimate (3.22) for the Krylov algorithm is still a little pessimistic. For example, from Figure 6 we see that using as a stopping criterion the estimate (3.22) yields about 25 iterations more than necessary.

\section{4. $2 D$ and 3D Finite Element Matrices}

As a second set of test cases we consider Richard's equation, which models fluid flow in groundwater. When the porous medium is homogeneous (i.e. $K=I$ ) and unsaturated, and we consider a vertical section, we get the diffusion equation (4.24) on a rectangular 2D 


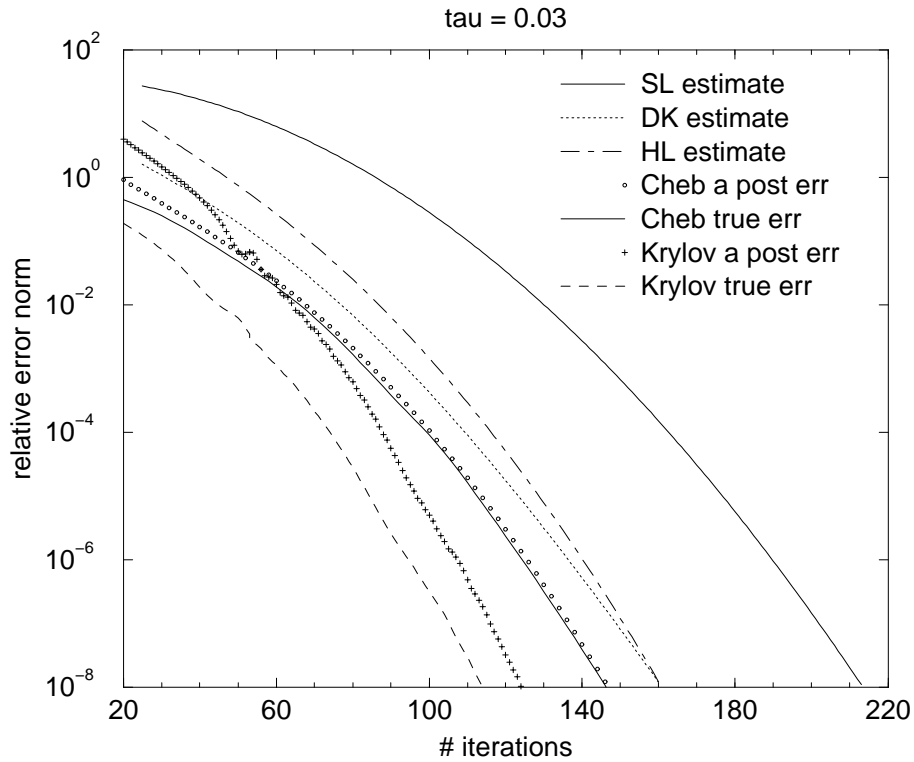

Figure 5. Comparison of Chebyshev and Krylov true errors with a priori and a posteriori estimates for the FD case with $s=60$ and $\tau=0.03$.

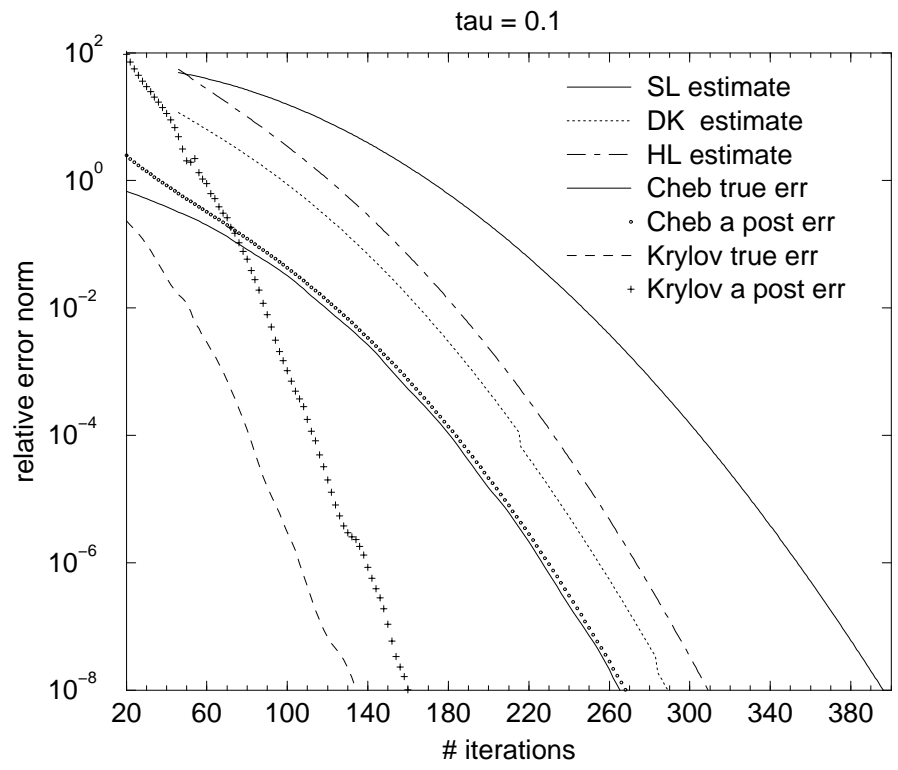

Figure 6. Comparison of Chebyshev and Krylov true errors with a priori and a posteriori estimates for the FD case with $s=60$ and $\tau=0.1$. 


\begin{tabular}{crrrrrr} 
mesh \# & nx & $\times$ & ny & \# of triang. & d.o.f. & nonzero \\
\hline 1 & 80 & $\times$ & 120 & 19,200 & 28,600 & 142,204 \\
2 & 160 & $\times$ & 240 & 76,800 & 114,800 & 572,404 \\
3 & 320 & $\times$ & 480 & 307,200 & 460,000 & $2,296,804$
\end{tabular}

Table 3. Main features of the three FE meshes in the 2D case.

\begin{tabular}{|c|c|c|c|c|c|c|c|c|c|}
\hline \multirow[b]{3}{*}{ mesh } & \multirow[b]{3}{*}{$\tau$} & \multirow[b]{3}{*}{$\lambda_{n}(\tau A)$} & \multicolumn{4}{|c|}{ Krylov } & \multicolumn{3}{|c|}{ Chebyshev } \\
\hline & & & & & $\mathrm{CP}$ & (s) & & CPL & \\
\hline & & & $\nu$ & \# it. & 1 st run & total & \# it. & total & eig \\
\hline \multirow{3}{*}{1} & 0.1 & 79 & 500 & 32 & 0.53 & 0.53 & 38 & 0.49 & 0.06 \\
\hline & 0.3 & 238 & 500 & 56 & 0.90 & 0.90 & 64 & 0.80 & 0.06 \\
\hline & 1.0 & 795 & 500 & 104 & 1.69 & 1.69 & 115 & 1.45 & 0.06 \\
\hline \multirow{3}{*}{2} & 0.1 & 300 & 121 & 66 & 5.20 & 5.20 & 72 & 5.74 & 0.31 \\
\hline & 0.3 & 901 & 121 & 114 & 9.00 & 9.00 & 123 & 10.03 & 0.31 \\
\hline & 1.0 & 3,002 & 121 & 210 & 17.06 & 23.61 & 225 & 17.42 & 0.31 \\
\hline \multirow{3}{*}{3} & 0.1 & 1,201 & 12 & 129 & 45.35 & 81.69 & $\overline{142}$ & 46.88 & 1.34 \\
\hline & 0.3 & 3,603 & 12 & 227 & 79.32 & 146.00 & 245 & 80.09 & 1.34 \\
\hline & 1.0 & 12,011 & 12 & 426 & 148.38 & 277.27 & 455 & 144.93 & 1.34 \\
\hline
\end{tabular}

Table 4. Number of iterations and CPU time for the Krylov and Chebyshev algorithms applied to the 2D-FE sample problems with $\epsilon=10^{-8}$. The last column of the Table accounts for the CPU time spent in the computation of the extreme eigenvalues.

domain. Discretization through Darcy's law by triangular Mixed Finite Element of lowest order $\left(\mathrm{RT}_{0}\right)$ generates a system of ODEs of the form (1.1), with a symmetric positive definite matrix $A$, having as many rows as the triangulation edges, and five nonzero elements per row [3]. Three regular nested grids are considered, whose features are described in Table 3.

The largest eigenvalue has been approximated using Lanczos method; only four iterations were needed to estimate $\lambda_{n}$ at the first significative digit, according to the convergence profiles in Figure 3. The smallest has been computed on a coarser mesh (say $10 \times 15$ ), at a completely negligible cost. This rough approximation does not significatively affect the number of iterations, as expected from Figure 2.

In Table 4 we compare the performance of Krylov and Chebyshev algorithms on the three FE meshes with $\tau=0.1,0.3,1.0$, and initial data $\boldsymbol{v}=\boldsymbol{y}_{0}=[1,1, \ldots, 1]^{T}$. Figure 7 displays the convergence profiles for the intermediate $160 \times 240$ mesh.

Finally, we consider a Galerkin Finite Element discretization of the flow equation described at the beginning of this Section, in an inhomogeneous porous medium, on the whole 3D domain [16]. This test case is characterized by high variations in the permeability tensor $K$, up to eight orders of magnitude, and thus a very fine mesh is needed to properly describe the flow field. Discretization is accomplished with about 1,5 million tetrahedral $\left(P_{1}\right)$ Finite Elements; the resulting matrix $A$ in the differential system (4.24) has 


\begin{tabular}{|rr|rrrr|rrr|}
\hline & & \multicolumn{4}{|c|}{ Krylov } & \multicolumn{3}{c|}{ Chebyshev } \\
& & \multicolumn{4}{|c|}{ CPU (s) } & & CPU (s) \\
$\tau$ & $\lambda_{n}(\tau A)$ & $\nu$ & \# it. & 1st run & total & \# it. & total & eig \\
\hline 0.01 & 96 & 38 & 36 & 10.54 & 10.54 & 44 & 13.17 & 1.87 \\
0.03 & 287 & 38 & 64 & 19.52 & 26.24 & 75 & 21.31 & 1.87 \\
0.1 & 956 & 38 & 118 & 34.71 & 55.34 & 135 & 37.11 & 1.87 \\
0.3 & 2870 & 38 & 215 & 61.93 & 107.55 & 234 & 63.07 & 1.87 \\
1.0 & 9563 & 38 & 395 & 114.34 & 206.31 & 426 & 113.16 & 1.87 \\
\hline
\end{tabular}

Table 5. Number of iterations and CPU time for the Krylov and Chebyshev algorithms applied to the 3D-FE sample problem with $\epsilon=10^{-8}$. The last column of the Table accounts for the CPU time spent in the computation of the extreme eigenvalues.

\section{Chebychev vs Krylov}

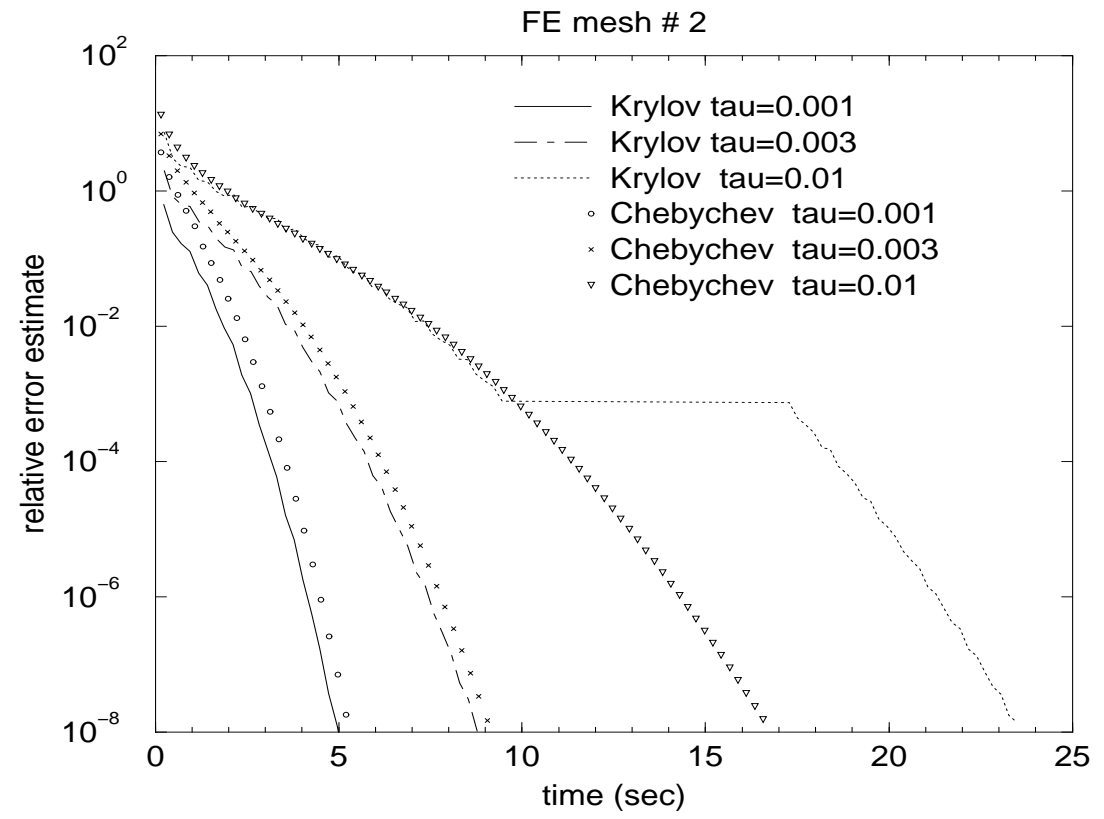

Figure 7. Relative error norm versus CPU time in the evaluation of $\exp (-\tau A) \boldsymbol{v}$ for the FE mesh \# 2 and for the three different values of $\tau$. The horizontal line accounts for the computation of the Lanczos coefficients (from $\nu+1$ to $m$ ) in the first Lanczos run.

$N=268,515$ rows and 3, 926, 214 non-zeros. In Table 5 we report the performance of Krylov and Chebyshev algorithms on the given FE mesh with $\tau=0.01,0.03,0.1,0.3,1.0$, and initial data $\boldsymbol{v}=\boldsymbol{y}_{0}=[1,1, \ldots, 1]^{T}$. In this case three Lanczos iterations were sufficient to estimate $\lambda_{n}$ at the first significative digit, while just two iterations of the Rayleigh Quotient procedure were enough to approximate the smallest eigenvalue with a relative error of $20 \%$ (recall Figures 2 and 3). 
Differently from the FD cases, we notice that in both 2D and 3D FE examples, the scaling of Krylov and Chebyshev algorithms with respect to $\tau$ is essentially the same. Indeed, the iteration numbers of the two methods are very close, and hence Chebyshev and Krylov performances are comparable (when the second run is not required). When the second Krylov run must be done, see e. g. mesh \#2 for $\tau=1.0$ and mesh \#3 for the 2D problem, and $\tau \geq 0.03$ for the 3D case, the Krylov cost increases up to almost twice the Chebyshev cost. We have also numerical evidence that convergence of Chebyshev method is driven only by the magnitude of the spectral radius of $\tau A$; compare close values of $\lambda_{n}(\tau A)$ in Tables 2, 4 and 5.

A final remark is in order: one could notice that the timestep values $\tau$ exhibited in the Tables lead to a large number of iterations for both methods. In these cases the advantage of our Chebyshev approach appears more pronounced. We stress that in the solution of the ODE system (1.1) the time-step usually increases moving away from the initial time and that, as documented e.g. in [9], one large step (when the problem allows for) is computationally more convenient than a sequence of smaller steps. Moreover, on very large size $3 \mathrm{D}$ problems, the number of storable Ritz vectors can be so small (cf. Tables 2 and 4) that a second Lanczos run is always required.

\section{Conclusions}

Our numerical tests show that Chebyshev series expansion can be considered a valid alternative to Krylov subspace method for the computation of the exponential operator, in the case of large, sparse, symmetric matrices. Such problems arise naturally after space discretization of 2D and 3D parabolic PDEs by Finite Differences or Finite Elements. The advantage of Chebyshev approach stems from its intrinsic simplicity, which allows an extremely efficient implementation with optimized storage allocation. In particular, on FE discretizations the number of iterations required by the Chebyshev and Krylov methods are very close, and thus the former approach is competitive even on relatively small problems, where the whole Krylov basis can be stored. We have also shown that the main drawback of the Chebyshev method, i.e. the necessity of estimating the spectral interval, does not affect significatively its performance on such problems.

These considerations lead naturally to further developments. First, Chebyshev approach could be conveniently pursued in the computation of other functions of large symmetric matrices. Consider, e.g., functions related to the matrix square root, which play a key role in the discretization of hyperbolic PDEs (as originally proposed in [5]). We quote also computation of exponential (or cosine) integrators [12] for large semi-linear ODEs systems, stemming from FD or FE discretization of 2D and 3D scalar reaction-diffusion (or semi-linear hyperbolic) equations, where Jacobian matrices are still symmetric.

Moreover, in the non-symmetric case, working with large Krylov subspaces produces a growing computational cost in addition to the growing storage required, since a longterm recurrence is involved, or a slower convergence when suitable restarts are employed. This suggests that Chebyshev and Faber polynomial methods could represent a practicable alternative to Krylov method even in non-symmetric instances, where estimation of the spectrum is much more difficult. In particular, when the spectrum is estimated by an ellipse (using the Arnoldi method for a rough approximation of the extremal eigenvalues), as it is well-known the Faber recurrence becomes the usual three-term Chebyshev recurrence, thus maintaining all the advantages described above in the symmetric case. Alternatively, if the 
spectrum is more generally approximated by a convex set, the long-term Faber recurrence can be truncated at a fixed length, saving convergence at a lower computational cost and storage requirement; see [15] for the first results in this direction and the references therein on Faber series.

\section{Acknowledgments}

This work has been supported in part by the MURST funds and by the GNIM-CNR. The first author has also been supported by EC contract \# IC15 CT96-211.

\section{REFERENCES}

1. M. Abramowitz and I. A. Stegun. Handbook of Mathematical Functions. Dover Publications, New York, NY, 1992.

2. L. Bergamaschi, G. Gambolati, and G. Pini. Asymptotic convergence of conjugate gradient methods for the partial symmetric eigenproblem. Numer. Lin. Alg. Appl., 4(2):69-84, 1997.

3. L. Bergamaschi and M. Putti. Mixed finite elements and Newton-like linearization for the solution of Richard's equation. Int. J. Numer. Methods Eng., 45(8):1025-1046, 1999.

4. V. L. Druskin, A. Greenbaum, and L. A. Knizhnerman. Using nonorthogonal Lanczos vectors in the computation of matrix functions. SIAM J. Sci. Comput., 19:38-54, 1998.

5. V. L. Druskin and L. A. Knizhnerman. Two polynomial methods for calculating functions of symmetric matrices. U.S.S.R. Comput. Math. and Math. Phys., 6:112-121, 1989.

6. V. L. Druskin and L. A. Knizhnerman. Krylov subspace approximation of eigenpairs and matrix functions in exact and computer arithmetics. Num. Lin. Alg. Appl., 2:205-217, 1995.

7. R. A. Friesner, L. S. Tuckerman, B. D. Dornblaser, and T. V. Russo. A method for exponential propagation of large systems of stiff nonlinear differential equations. J. Sci. Comput., 4:327354, 1989.

8. E. Gallopoulos and Y. Saad. In Proc. 1989 ACM International Conference on Supercomputing, pages $17-28,1989$.

9. E. Gallopoulos and Y. Saad. Efficient solution of parabolic equations by Krylov subspace methods. SIAM J. Sci. Stat. Comput., 13(5):1236-1264, 1992.

10. G. H. Golub and C. F. van Loan. Matrix Computation. Johns Hopkins University Press, Baltimore, 1991.

11. M. Hochbruck and J. J. Lubich. On Krylov subspace approximations to the matrix exponential. SIAM J. Numer. Anal., 34(5):1911-1925, 1997.

12. M. Hochbruck, J. J. Lubich, and H. Selhofer. Exponential integrators for large systems of differential equations. SIAM J. Sci. Comput., 19:1552-1574, 1998.

13. L. A. Knizhnerman. Calculation of functions of unsymmetric matrices using Arnoldi's method. Comput. Math. and Math. Phys., 31:1-9, 1991.

14. G. Meinardus. Approximation von Funktionen und ihre numerische Behandlung. SpringerVerlag, 1964.

15. I. Moret and P. Novati. An interpolatory approximation of the matrix exponential based on Faber polynomials. 1999. (to appear).

16. C. Paniconi and M. Putti. A comparison of Picard and Newton iteration in the numerical solution of multidimensional variably saturated fbw problems. Water Resour. Res., 30(12):3357-3374, 1994.

17. Y. Saad. Analysis of some Krylov subspace approximations to the matrix exponential operator. SIAM J. Numer. Anal., 29(1):209-228, 1992.

18. R. B. Sidje. EXPOKIT: Software package for computing matrix exponentials. ACM Trans. Math. Software, 24:130-156, 1998.

19. D. E. Stewart and T. S. Leyk. Error estimates for Krylov subspace approximations of matrix exponentials. J. Comput. Applied Math., 72:359-369, 1996.

20. H. Tal-Ezer. Spectral methods in time for parabolic problems. SIAM J. Numer. Anal., 26:1-11, 1989. 